\title{
Choosing a healthy breakfast cereal - granola takes the biscuit
}

\author{
T.T. White-Flynn ${ }^{1}$, B.J. Kemp ${ }^{1}$, B.E. Cronin ${ }^{1}$, O.C. Lyons ${ }^{1,2}$, O. Bruton ${ }^{1}$, C.B. O’Donovan ${ }^{1}$, \\ C.M. Donovan ${ }^{1}$ and M.A.T. Flynn ${ }^{1,2}$ \\ ${ }^{1}$ Food Safety Authority of Ireland, Dublin 1, Republic of Ireland and \\ ${ }^{2}$ Northern Ireland Centre for Food and Health, University of Ulster, Coleraine, BT52 1SA, UK
}

High fibre breakfast cereals which are low in fat, saturated fat and sugar are recommended for healthy eating in Ireland ${ }^{(1)}$. Making healthy choices can be challenging given the wide variety of breakfast cereals available on the Irish market. However, cut-off criteria established for making nutrition claims for total fat, saturated fat, sugar and fibre, can be used to identify such healthy choices ${ }^{(2)}$. The aim of this study was to explore the application of nutrition claims criteria to all breakfast cereals available on the market.

Data was collected on breakfast cereals available in Ireland between July 2016 and March 2017 including brand/name of product, macronutrient information, portion size and presence of nutrition and health claims. Breakfast cereals $(n$ 453) were categorised into 5 groups; oats ( $n$ 112), compressed biscuit (CB) $(n$ 45), flake/puffed ( $n$ 154), muesli $(n$ 69) and granola $(n$ 73). Based on nutrition claim legislation $^{(2)}$, healthy choice $(\mathrm{HC})$ criteria for total fat $(\leqslant 3 \mathrm{~g})$, saturated fat $(\leqslant 1.5 \mathrm{~g})$ and fibre $(\geqslant 3 \mathrm{~g})$ were adopted on a per portion basis. For sugar, a range of cut-offs $(4-8 \mathrm{~g})$ was examined, with $\leqslant 6 \mathrm{~g}$ per portion emerging as the most appropriate. The categories of cereals meeting the healthy choice $(\mathrm{HC})$ criteria and others that did not $(\mathrm{O})$ were compared, see table below.

\begin{tabular}{|c|c|c|c|c|c|c|c|}
\hline Cereal categories & & $\begin{array}{c}\mathrm{HC} \mathrm{/} \mathrm{O} \\
\%(n)\end{array}$ & $\begin{array}{l}\text { Energy } \\
\text { (Kcal) }\end{array}$ & $\begin{array}{l}\text { Total Fat } \\
(\mathrm{g})\end{array}$ & $\begin{array}{c}\text { Sat Fat } \\
(\mathrm{g})\end{array}$ & $\begin{array}{l}\text { Sugar } \\
(\mathrm{g})\end{array}$ & $\begin{array}{c}\text { Fibre } \\
(\mathrm{g})\end{array}$ \\
\hline \multirow[t]{2}{*}{ Oats } & $\mathrm{HC}$ & $30(34)$ & $\begin{array}{l}148 \cdot 4 \\
(76.6-199.7)\end{array}$ & $\begin{array}{l}2 \cdot 4 \\
(0 \cdot 8-3 \cdot 0)\end{array}$ & $\begin{array}{l}0.4 \\
(0 \cdot 1-0 \cdot 6)\end{array}$ & $\begin{array}{l}0 \cdot 5 * * \\
(0 \cdot 0-5 \cdot 2)\end{array}$ & $\begin{array}{l}3 \cdot 8 * * * \\
(3 \cdot 0-6 \cdot 8)\end{array}$ \\
\hline & $\mathrm{O}$ & $70(78)$ & $\begin{array}{l}144 \cdot 0 \\
(89 \cdot 2-281 \cdot 3)\end{array}$ & $\begin{array}{l}2 \cdot 5 \\
(0 \cdot 9-5 \cdot 3)\end{array}$ & $\begin{array}{l}0 \cdot 4 \\
(0 \cdot 0-4 \cdot 6)\end{array}$ & $\begin{array}{l}1 \cdot 6 \\
(0 \cdot 2-18 \cdot 2)\end{array}$ & $\begin{array}{l}2 \cdot 9 \\
(0 \cdot 9-6 \cdot 3)\end{array}$ \\
\hline \multirow[t]{2}{*}{$\mathrm{CB}$} & $\mathrm{HC}$ & $36(16)$ & $\begin{array}{l}139 \cdot 2 * \\
(134 \cdot 3-163 \cdot 4)\end{array}$ & $\begin{array}{l}0.8 * * \\
(0 \cdot 7-1 \cdot 0)\end{array}$ & $\begin{array}{l}0 \cdot 2^{*} \\
(0 \cdot 1-0 \cdot 2)\end{array}$ & $\begin{array}{l}1 \cdot 7 * * * \\
(0 \cdot 3-5 \cdot 9)\end{array}$ & $\begin{array}{l}3 \cdot 8 * * * \\
(3 \cdot 6-5 \cdot 2)\end{array}$ \\
\hline & $\mathrm{O}$ & $64(29)$ & $\begin{array}{l}152 \cdot 0 \\
(112 \cdot 5-234 \cdot 5)\end{array}$ & $\begin{array}{l}1.9 \\
(0 \cdot 6-9 \cdot 5)\end{array}$ & $\begin{array}{l}0 \cdot 4 \\
(0 \cdot 1-2 \cdot 8)\end{array}$ & $\begin{array}{l}8 \cdot 4 \\
(0 \cdot 9-12 \cdot 4)\end{array}$ & $\begin{array}{l}3 \cdot 3 \\
(1 \cdot 5-4 \cdot 5)\end{array}$ \\
\hline \multirow[t]{2}{*}{ Flake/Puffed } & $\mathrm{HC}$ & $10(15)$ & $\begin{array}{l}120 \cdot 0 \\
(84 \cdot 0-164 \cdot 3)\end{array}$ & $\begin{array}{l}0 \cdot 9 \\
(0 \cdot 2-2 \cdot 4)\end{array}$ & $\begin{array}{l}0 \cdot 1 \\
(0 \cdot 0-0 \cdot 3)\end{array}$ & $\begin{array}{l}4 \cdot 2 \\
(0 \cdot 5-6 \cdot 0)\end{array}$ & $\begin{array}{l}3 \cdot 9 * * * \\
(3 \cdot 0-5 \cdot 5)\end{array}$ \\
\hline & $\mathrm{O}$ & 90 (139) & $\begin{array}{l}114 \cdot 9 \\
(90 \cdot 0-204 \cdot 3)\end{array}$ & $\begin{array}{l}0 \cdot 8 \\
(0 \cdot 1-11 \cdot 5)\end{array}$ & $\begin{array}{l}0 \cdot 2 \\
(0 \cdot 0-6 \cdot 8)\end{array}$ & $\begin{array}{l}5 \cdot 7 \\
(0-12 \cdot 8)\end{array}$ & $\begin{array}{l}1 \cdot 5 \\
(0 \cdot 3-10 \cdot 8)\end{array}$ \\
\hline \multirow[t]{2}{*}{ Muesli } & $\mathrm{HC}$ & $9(6)$ & $\begin{array}{l}155 \cdot 5 \\
(120 \cdot 0-174 \cdot 2)\end{array}$ & $\begin{array}{l}2 \cdot 1 * * \\
(1 \cdot 0-2 \cdot 7)\end{array}$ & $\begin{array}{l}0 \cdot 3 * * \\
(0 \cdot 2-0 \cdot 5)\end{array}$ & $\begin{array}{l}3 \cdot 5 * * * \\
(0 \cdot 5-5 \cdot 9)\end{array}$ & $\begin{array}{l}4 \cdot 1 \\
(3 \cdot 0-5 \cdot 0)\end{array}$ \\
\hline & $\mathrm{O}$ & $91(63)$ & $\begin{array}{l}170 \cdot 1 \\
(101 \cdot 1-241 \cdot 5)\end{array}$ & $\begin{array}{l}3 \cdot 9 \\
(0 \cdot 8-14 \cdot 0)\end{array}$ & $\begin{array}{l}0 \cdot 8 \\
(0 \cdot 1-2 \cdot 8)\end{array}$ & $\begin{array}{l}9 \cdot 5 \\
(3 \cdot 5-15 \cdot 3)\end{array}$ & $\begin{array}{l}3 \cdot 7 \\
(1 \cdot 1-6 \cdot 3)\end{array}$ \\
\hline Granola & $\mathrm{O}$ & $100(73)$ & $\begin{array}{l}199 \cdot 8 \\
(112 \cdot 7-250 \cdot 5)\end{array}$ & $\begin{array}{l}8 \cdot 3 \\
(3 \cdot 3-20 \cdot 6)\end{array}$ & $\begin{array}{l}1 \cdot 9 \\
(0 \cdot 3-11 \cdot 4)\end{array}$ & $\begin{array}{l}8 \cdot 1 \\
(1 \cdot 2-18 \cdot 6)\end{array}$ & $\begin{array}{l}3 \cdot 2 \\
(1 \cdot 0-6 \cdot 7)\end{array}$ \\
\hline
\end{tabular}

Values are presented as medians (minimum and maximum) per portion. Differences between categories were investigated using Mann-Whitney U Test where significance denoted as ${ }^{*} \mathrm{P}, 0.05, * * \mathrm{P}, 0 \cdot 01,{ }^{*} * \mathrm{P}, 0.001$. HC, Healthy Choice cereals i.e. those cereals meeting the healthy cereal criteria; $\mathrm{O}$, Other cereals i.e. those cereals who do not meet the healthy cereal criteria; $\mathrm{CB}$, compressed biscuit.

Applying the $\mathrm{HC}$ criteria, none of the granola cereals were considered healthy choices. All cereals meeting the $\mathrm{HC}$ criteria had higher fibre with the exception of muesli; all cereals meeting the HC criteria had lower sugar with the exception of flake/puffed categories. Only CB and muesli categories meeting HC criteria had significantly lower total and saturated fat. Significantly more products not meeting criteria for $\mathrm{HC}$ carried claims in the oats $(\mathrm{P}=0.036)$ and flaked/puffed $(\mathrm{P}=0.034)$ categories. Oats emerged as the category with the lowest median sugar content with a median fibre content of $3.8 \mathrm{~g}$.

This study shows that nutrient profiles based on portion size and nutrient cut-off criteria established for making nutrition claims, can be applied to breakfast cereals to identify healthy choices for consumers. Without nutrient profile criteria that are based on portion size, nutrition and health claims may mislead consumers as they are based on $100 \mathrm{~g}$. The approach used in this study could be developed and applied to guide consumers on healthy choices for other food categories.

1. Healthy Ireland (2016) The Food Pyramid. www.safefood.eu/Healthy-Eating/What-is-a-balanced-diet/The-Food-Pyramid.aspx (Accessed January 2017.)

2. European Commission (2006) Regulation No 1924/2006 on Nutrition and Health Claims made on food. www.fsai.ie/uploadedFiles/ Cor_Reg1924_2006(1).pdf (Accessed March 2017). 